WP ECON 17.04

\title{
Health and fairness with other-regarding preferences
}

Aitor Calo-Blanco

Departamento de Economía. Universidade da Coruña

Keywords: Health, Fairness, Other-Regarding Preferences, Social Ordering Function

J EL Classification: D62, D63, D71, I14 


\title{
Health and fairness with other-regarding preferences* $^{*}$
}

\author{
Aitor Calo-Blanco ${ }^{\dagger}$ \\ Universidade da Coruña
}

February 28, 2017

\begin{abstract}
We explore how to construct social preferences when individuals have other-regarding preferences over health and consumption. Our social evaluation also incorporates a view that aims to reduce inequalities that originate from factors for which individuals should not be deemed responsible.

Keywords: Health, Fairness, Other-Regarding Preferences, Social Ordering Function.

JEL classification: D62, D63, D71, I14.
\end{abstract}

\section{Introduction}

Behavioural economists have drawn attention to the importance of other-regarding preferences (ORPs) in the evaluation of individual well-being (see Fehr and Schmidt 2006). Unlike standard self-centered preferences, ORPs consider that individuals care about their own situation as well as others' (e.g., Fleurbaey 2012). In a recent paper, Decerf and Van der Linden (2016) examine how basic fairness and efficiency principles determine the construction of social preferences when agents have heterogeneous ORPs (see also Treibich 2015).

${ }^{*}$ I would like to thank Rafael Treibich, Mar Calo and the participants to seminars and conferences in Seville, Odense and Bilbao for their comments. Financial support from the Spanish Ministry of Economy and Competitiveness (ECO2011-22919 and ECO201457413-P) is gratefully acknowledged. The usual disclaimer applies.

${ }^{\dagger}$ Corresponding author at: Departamento de Economía, Universidade da Coruña, Campus de A Coruña, 15071 A Coruña, Spain. E-mail: aitorcalo@gmail.com. 
The aim of this note is to extend this last approach with the inclusion of individuals who care about health and who may differ in both their initial resources and their health care needs. There are, at least, two important reasons to focus our extension on a good such as health. On the one hand, as Fleurbaey and Schokkaert (2011) emphasise, the individual trade-off between health and other goods presents a distinctive feature because the level of health strongly conditions one's own well-being evaluation. To deal with this issue, equity and fairness axioms that aim to enhance social welfare have to be anchored to a particular reference point. Fleurbaey (2005) proposes to resort to the state of perfect health since at this reference level the individual well-being can be measured exclusively in terms of consumption, regardless of what the individual preferences are. On the other hand, there is evidence that a person's health state may affect the well-being of any nearby individual (e.g., Bruhin and Winkelmann 2009), and hence ORPs should be relevant for the social evaluation of any health care policy.

This second reason raises a crucial issue at the time of evaluating social welfare when agents have ORPs. As Decerf and Van der Linden (2016) point out, the resulting social ranking will depend on the extent of the otherregarding part of the individual preferences that the planner is willing to use. In this note we consider that the planner will take such a part into account at the time of designing both efficiency and fairness axioms. The case of efficiency is easy to justify as it seems natural to promote changes that make no-one worse-off. By contrast, fairness principles that compensate individuals for their ORPs normally generate further controversy, as they may benefit agents with envious preferences. However, in the case of health these principles can be more easily justified as the effect of others' health in one's own well-being is not always necessarily fairly distributed among all agents. For instance, Braakmann (2014) finds that in Germany women, but not men, suffer significant well-being losses from 'spousal disability'. This implies that social norms may promote, in a framework with health, heterogeneous other-regarding views which are worth compensating. Another example is provided by Kerschbamer et al. (2016), who show that standard solutions in credence markets, like the one for health care services, are not robust to the inclusion of individuals with ORPs. Specifically, when the quality of the service cannot (respectively, can) be verified, doctors with prosocial (anti-social) ORPs tend to reduce (increase) the expected outcomes of 'undertreatment' and 'overcharging'. Therefore, we adopt an ethical position which considers that relative views matter for equality. We do so by assuming a so-called non-resourcist approach in which a specific welfare dominance criterion is used to determine the extent of the equality principles (see Decerf and Van der Linden 2016). 


\section{The framework}

Our framework follows the fair social choice approach developed by Fleurbaey and Maniquet (2011). Let us consider a group of individuals $N=\{1, \ldots, n\}$ who care about two goods, consumption, $c \in \mathbb{R}_{+}$, and health, $h \in H=[0,1]$. Let $h^{*}:=1$ denote the state of perfect health. Every individual $i \in N$ is endowed with an amount of initial resources $\omega_{i} \in \mathbb{R}_{++}$ that she devotes to both consumption and medical expenditure. Let $\omega_{N}=\left(\omega_{1}, \ldots, \omega_{n}\right)=\left(\omega_{i}\right)_{i \in N} \in \mathbb{R}_{++}^{n}$ be the profile of resources, and $\Omega=\sum_{i \in N} \omega_{i}$ be the social endowment of these resources in this society. Agent $i \in N$ is also characterised by her medical disposition $m_{i} \in \mathbb{R}_{++}$, which defines the amount of medical expenditure $m_{i} h \in \mathbb{R}_{+}$that she needs to invest in order to reach a given health state $h \in H$. Let $\mathcal{M}=\left[m^{-}, m^{+}\right]$ be the set of all the feasible medical dispositions, where $m^{-}$and $m^{+}$ determine, respectively, the best and the worst possible ones, and hence $m^{-}<m^{+}$. The population's profile of health dispositions is described by $m_{N}=\left(m_{i}\right)_{i \in N} \in \mathcal{M}^{n}$. The extreme values of $\mathcal{M}$, as well as the social endowment, are assumed to be fixed for all possible allocations.

In health models it is usually assumed that only income and expenditure are observable, and hence the health state is considered to be agents' private information (e.g., Valletta 2014). Therefore, since we have already established that individuals care about others' situation, let us now introduce an observable space that consists of consumption and medical expenditure. In this scenario each individual $i \in N$ has a consumption-expenditure bundle $x_{i}=\left(c_{i}, e_{i}\right) \in X=\mathbb{R}_{+}^{2}$. Such a pair designates the situation in which she has a level of consumption $c_{i}$ and a medical expenditure $e_{i}=m_{i} h_{i}$, which consists of the amount of resources that she must pay to reach a health state $h_{i}$ given her fixed medical disposition $m_{i}$. Consequently, when the value of $e_{i}$ is equal to $m_{i}$ the individual gets the state of perfect health. An allocation $x_{N}=\left(x_{i}\right)_{i \in N} \in X^{n}$ describes all the individuals' bundles. Let $R_{i}^{m_{i}}$ denote agent $i$ 's preferences over this observable set of allocations $X^{n}$. Note that the preferences must also display the individual health care needs $m_{i}$ because agents may transform their medical expenditure into health in different ways. $x_{N} R_{i}^{m_{i}} x_{N}^{\prime}$ means that individual $i$ weakly prefers $x_{N}$ to allocation $x_{N}^{\prime}$. Strict preference and indifference relations are denoted by $P_{i}^{m_{i}}$ and $I_{i}^{m_{i}}$ respectively. A profile of ORPs preferences is denoted by $R_{N}=\left(R_{i}^{m_{i}}\right)_{i \in N}$.

Since ORPs excessively widen the domain of admissible individual preferences, we introduce some simplifications. First, ORPs are assumed to be a complete preorder (a binary relation that is reflexive, transitive and complete), continuous and strictly monotonic when others' bundle remain 
constant. Second, ORPs satisfy the following properties:

ORPs Axiom 1 (Separability) For all $x_{N}, x_{N}^{\prime} \in X^{n}$ and $i \in N$ :

$$
\left(x_{i}, x_{N \backslash\{i\}}\right) R_{i}^{m_{i}}\left(x_{i}^{\prime}, x_{N \backslash\{i\}}\right) \Leftrightarrow\left(x_{i}, x_{N \backslash\{i\}}^{\prime}\right) R_{i}^{m_{i}}\left(x_{i}^{\prime}, x_{N \backslash\{i\}}^{\prime}\right),
$$

where $x_{N \backslash\{i\}}=\left(x_{1}, \ldots, x_{i-1}, x_{i+1}, \ldots, x_{n}\right)$.

ORPs Axiom 2 (Consumption Desirability) For all $x_{N}, x_{N}^{\prime} \in X^{n}, i \in N$, $c>0$ and $e, e^{\prime} \in \mathbb{R}_{+}$:

$$
\left((c, e), x_{N \backslash\{i\}}\right) P_{i}^{m_{i}}\left(\left(0, e^{\prime}\right), x_{N \backslash\{i\}}^{\prime}\right) .
$$

Separability means that the individual ordinal evaluation of one's own choice does not depend on the others' choices. Hence, for any $i \in N$ we can associate $R_{i}^{m_{i}}$ with a unique set of internal preferences $\widehat{R}_{i}^{m_{i}}$ over the set of bundles $X$, where $x_{i} \widehat{R}_{i}^{m_{i}} x_{i}^{\prime}$ means that individual $i$ weakly prefers bundle $\left(c_{i}, e_{i}\right)$ to bundle $\left(c_{i}^{\prime}, e_{i}^{\prime}\right)$. These preferences are continuous, convex, strictly monotonic and a complete preorder. The asymmetric and symmetric parts of $\widehat{R}_{i}^{m_{i}}$ are denoted by $\widehat{P}_{i}^{m_{i}}$ and $\widehat{I}_{i}^{m_{i}}$ respectively. Note that from this internal perspective there is a one-to-one correspondence between the consumption-health and the consumption-expenditure spaces. This is so because the individual medical disposition is fixed, and hence a given amount of medical expenditure uniquely determines a given health state. Consumption Desirability sets a limit to the externalities that anyone may suffer. Specifically, it considers that no agent prefers a situation without consumption whatever the others have. This view that life cannot be enjoyed without consumption is defended by Fleurbaey and Schokkaert (2011).

Additionally, we restrict the admissible profile of ORPs in this society by assuming that there exist both altruistic and envious agents, as well as self-centered individuals. Formally,

Profile Axiom 1 (Plurality of Preferences) For all $x_{N}, x_{N}^{\prime} \in X^{n}$, if $x_{i}^{\prime}>x_{i}{ }^{1}$ for some $i \in N$ and $x_{N \backslash\{i\}}^{\prime}=x_{N \backslash\{i\}}$, then there exist $j, k, q \neq i$ such that:

$$
x_{N} R_{j}^{m_{j}} x_{N}^{\prime}, \quad x_{N}^{\prime} R_{k}^{m_{k}} x_{N} \text { and } x_{N} I_{q}^{m_{q}} x_{N}^{\prime} .
$$

Let $\mathcal{R}$ denote the domain of profiles of ORPs that satisfy all these properties, and hence $R_{N} \in \mathcal{R}$.

An economy is then described by a list $E=\left(\omega_{N}, m_{N}, R_{N}\right) \in \mathcal{E}$, where $\mathcal{E}$ is the domain of all the economies that satisfy the previous assumptions. A

\footnotetext{
${ }^{1}$ Vector inequalities are denoted $\geq,>, \gg$.
} 
social ordering function, SOF hereafter, $\mathbf{R}(E)$ defines a complete order over all allocations. For any $E \in \mathcal{E}$ and $x_{N}, x_{N}^{\prime} \in X^{n}$, let $x_{N} \mathbf{R}(E) x_{N}^{\prime}$ denote that allocation $x_{N}$ is at least as good as $x_{N}^{\prime}$. Strict preference is denoted by $\mathbf{P}(E)$, and the indifference relation by $\mathbf{I}(E)$.

We now introduce some basic definitions that will be useful to present our result. Let us start with the set of all the bundles that an individual can afford:

Definition 1 For all $E \in \mathcal{E}$ and $i \in N$, the individual $i$ 's consumptionexpenditure feasible set is:

$$
B\left(\omega_{i}\right)=\left\{(c, e) \in X \mid c+e \leq \omega_{i}\right\} .
$$

Second, we define a measure of individual well-being for the case of self-centered preferences (see Fleurbaey 2005). This value is the smallest consumption that an individual would accept to exchange her actual bundle for one in which she has perfect health. Note that such a value depends on the way in which the individual transforms her medical expenditure into health:

Definition 2 For all $E \in \mathcal{E}, i \in N$ and $x_{i} \in X$, the individual $i$ 's full-health equivalent consumption (FHEC) is the value $c_{i}^{m_{i}}\left(x_{i}\right)$ that satisfies:

$$
c_{i}^{m_{i}}\left(x_{i}\right)=\min \left\{c^{\prime} \in \mathbb{R}_{+} \mid\left(c^{\prime}, m_{i}\right) \widehat{R}_{i}^{m_{i}}\left(c_{i}, e_{i}\right)\right\} .
$$

Let us now present a hypothetical situation in which an individual would be maximising her utility, according to her internal preferences, if she had the best medical disposition and an equal split of the social endowment $\Omega$. The reason why we propose this hypothetical point is twofold. First, given that it is defined in terms of internal preferences this hypothetical situation can be interpreted as a scenario in which the individual is free of any possible externality. Second, since this point is anchored to both the best medical disposition and the average social endowment, it allows us to introduce a concern for inequality of opportunity in terms of health care needs and initial resources. Formally,

Definition 3 For all $E \in \mathcal{E}$ and $i \in N$, the individual $i$ 's best equal-split bundle is the pair $\bar{x}_{i} \in X$ that satisfies:

$$
\left.\bar{x}_{i} \in \max \right|_{\widehat{R}_{i}^{m-}} B(\bar{\omega}),
$$

where $\bar{\omega}=\frac{\Omega}{|N|}$ is an equal split of the social endowment. 
Finally, we introduce our measure of individual well-being. This measure relates any individual's situation to a proportion of the FHEC associated with her best equal-split bundle, specifically, to the proportion that would make her indifferent between the actual allocation and a hypothetical ideal situation. In such a situation all individuals would be endowed with the best medical disposition, and they would have both perfect health and the FHEC associated with their own specific best equal-split bundle:

Definition 4 For all $E \in \mathcal{E}, x_{N} \in X^{n}$ and $i \in N$, the individual i's FHEC equal-split equivalent is the scalar $\lambda_{i}\left(x_{N}\right) \in \mathbb{R}_{+}$that satisfies:

$$
x_{N} I_{i}^{m_{i}}\left(\left(\lambda_{i}\left(x_{N}\right) c_{i}^{m^{-}}\left(\bar{x}_{i}\right), m_{i}\right),\left(c_{j}^{m^{-}}\left(\bar{x}_{j}\right), m_{j}\right)_{j \in N \backslash\{i\}}\right) .
$$

In settings with ORPs it is not always possible to ensure that this sort of equivalent measure exists and is unique, since its value may not be defined for those who exhibit extreme cases of other-regarding views. For instance, the well-being of one of those agents might drastically change when the others' situations varied significantly, and hence her equivalent measure of well-being might go to infinity. However, as the following proposition states, in our framework this is an extreme possibility that might occur only for positive values, and only for a subgroup of agents. More precisely, apart from presenting a lower bound, the measure of individual well-being $\lambda_{i}\left(x_{N}\right)$ is always well-defined for a subset of the population:

Proposition 1 On the domain $\mathcal{E}$, the individual i's FHEC equal-split equivalent presents a non-negative lower bound. Moreover, for a non-empty subset of the population the value of this equivalent is finite and unique.

Let us now present a graphical illustration of this concept of FHEC equal-split equivalent $\lambda_{i}\left(x_{N}\right)$. Figure 1 depicts an economy with only two agents, $j$ and $k$, such that $\omega_{j}<\omega_{k}$ and $m_{j}>m_{k}$. Since ORPs are defined in a space with several dimensions, we focus on the individual internal preferences. Note that for any individual $i \in N$ it is not possible to get additional utility from the medical expenditure that is above $m_{i}$. Consequently, from this point onwards the internal indifference curves become completely flat showing, then, that additional health expenditure would not increase the level of utility. According to the initial conditions of the present example we have that individual $j$ has a smaller set of opportunities than $k$. Therefore, agent $j$ 's actual FHEC, $c_{j}^{m_{j}}\left(x_{j}\right)$ (see the black solid curve), is smaller than the one associated with her best 


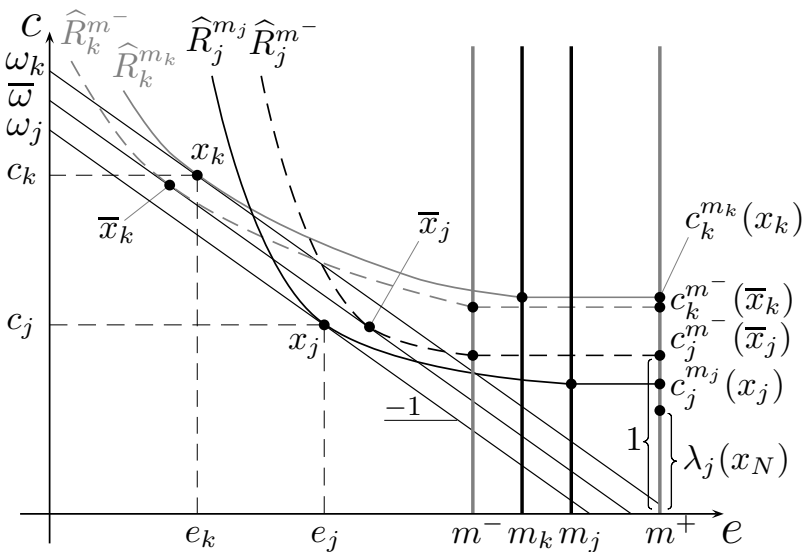

Figure 1: The FHEC equal-split equivalent

equal-split bundle, $c_{j}^{m^{-}}\left(\bar{x}_{j}\right)$, which is obtained with the hypothetical conditions (see the black dashed curve). This is so because the agent is endowed with both a bad medical disposition and fewer resources than an equal split of the social endowment. Note that this assessment of individual well-being loss is exclusively related to an internal viewpoint. As regards the relative views, we can consider that individual $j$ experiences a utility loss from observing that agent $k$ enjoys a higher share of the social endowment. As a result of this, $j$ 's actual equivalent utility would not be described by $c_{j}^{m_{j}}\left(x_{j}\right)$, but by a smaller amount of consumption. More precisely, $j$ considers the social allocation to be equivalent to a scenario in which she both was alone in the economy and had a bundle that entailed a FHEC equal to $\lambda_{j}\left(x_{N}\right) c_{j}^{m^{-}}\left(\bar{x}_{j}\right)<c_{j}^{m_{j}}\left(x_{j}\right)$ (see Figure 1). This relation describes that individual $j$ suffers a negative externality from observing that $k$ has a higher level of total expenditure than her.

\section{$3 \quad$ Ethical axioms and social preferences}

We will start this section by introducing the ethical axioms that our society endorses. Next, we will use such principles to construct our SOF.

The first ethical axiom is a requirement which ensures, given the individuals' ORPs, that the solution is efficient:

SOF Axiom 1 (Strong Pareto): For all $E \in \mathcal{E}$ and $x_{N}, x_{N}^{\prime} \in X^{n}$, if $x_{N} R_{i}^{m_{i}} x_{N}^{\prime}$ for all $i \in N$; then $x_{N} \mathbf{R}(E) x_{N}^{\prime}$. If moreover, $x_{N} P_{j}^{m_{j}} x_{N}^{\prime}$ for some $j \in N$; then $x_{N} \mathbf{P}(E) x_{N}^{\prime}$. 
In order to describe social preferences we need to combine Strong Pareto with axioms that model transfers. As we have previously stated, in the framework that we are analysing ORPs are assumed to be relevant in terms of equality. Consequently, our fairness axioms aim to reduce well-being differences that take into account the agents' relative views over the social allocation, something that we do by means of a particular well-being dominance criterion. For instance, our second principle establishes that a transfer of resources that reduces inequality between someone who is better-off than in the allocation characterised by the best equal-split situations and another agent who is worse-off than in this hypothetical allocation cannot reduce social welfare, provided that both keep their own preference relation with respect to such a hypothetical situation. Additionally, to respect the relative views of those who are not involved in the transfer they must remain indifferent between the pre- and the post-transfer allocations. The aim of this axiom is to provide those who have an excessively low well-being with a sort of 'safety net'.

SOF Axiom 2 (Equal-Split Equivalent Solidarity) For all $E \in \mathcal{E}, x_{N}, x_{N}^{\prime} \in$ $X^{n}$ and $\Delta \in \mathbb{R}$, if there exist $j, k \in N$ such that,

$$
\begin{gathered}
c_{j}+e_{j}=c_{j}^{\prime}+e_{j}^{\prime}-\Delta, \\
c_{k}+e_{k}=c_{k}^{\prime}+e_{k}^{\prime}+\Delta, \\
x_{N}^{\prime} P_{j}^{m_{j}} x_{N} P_{j}^{m_{j}}\left(c_{i}^{m^{-}}\left(\bar{x}_{i}\right), m_{i}\right)_{i \in N}, \\
\left(c_{i}^{m^{-}}\left(\bar{x}_{i}\right), m_{i}\right)_{i \in N} P_{k}^{m_{k}} x_{N} P_{k}^{m_{k}} x_{N}^{\prime},
\end{gathered}
$$

with $x_{N} I_{i}^{m_{i}} x_{N}^{\prime}$ for all $i \neq j, k$; then $x_{N} \mathbf{R}(E) x_{N}^{\prime}$.

This SOF axiom exhibits two important characteristics that are worth emphasising. On the one hand, the transfer need not be strictly positive as ORPs may entail that an individual is better-off (conversely, worse-off) after paying (receiving) some resources. On the other hand, the axiom is defined in terms of the relative view with respect to the FHEC equal-split equivalent bundle, which is constructed by means of the best medical disposition and the average resources in the society. Therefore, Equal-Split Equivalent Solidarity also shows a concern for those who have less than an equal split of the total resources and/or a very low medical disposition, fully exploiting this way the agents' heterogeneity. Nevertheless, although this fairness principle addresses the differences that originate from factors which are beyond the agents' responsibility, the way in which these differences are compensated is limited by the preference relation with respect to the hypothetical allocation $\left(c_{i}^{m^{-}}\left(\bar{x}_{i}\right), m_{i}\right)_{i \in N}$. 
To strengthen compensations for differences in initial resources and/or health care needs, let us introduce an additional transfer axiom that allows us to deal separately with those individuals who share their other-regarding views. Specifically, we propose a slight variant of Equal-Split Equivalent Solidarity that only applies to those who have identical ORPs for all possible medical dispositions. That is, to those individuals who would behave exactly in the same way whenever they were endowed with the same medical disposition. This condition on the individual preferences, which is fairly demanding, permit us to avoid clashes with the other two ethical principles. As it was the case with the previous SOF axiom, this new welfare-enhancing transfer cannot reverse the well-being positions of the agents who are involved in it. Since they have identical other-regarding views, such positions can be defined by means of the level of consumption that would left an agent indifferent between the actual situation and the perfect health allocation in which any other agent $i$ would consume $c_{i}^{m^{-}}\left(\bar{x}_{i}\right)$. Then, the individual who is worse-off (conversely, better-off) after the transfer must remain better-off (worse-off) than in this hypothetical allocation when obtaining the monetary resources that the other agent would have in her own respective hypothetical situation. Formally,

SOF Axiom 3 (Equal Relative Views Solidarity) For all $E \in \mathcal{E}, x_{N}, x_{N}^{\prime} \in$ $X^{n}$ and $\Delta \in \mathbb{R}$, if there exist $\widehat{c}, \widetilde{c} \in \mathbb{R}_{++}, j, k \in N$ such that $R_{j}^{m}=R_{k}^{m}$ for all $m \in \mathcal{M}$, and moreover,

$$
\begin{gathered}
c_{j}+e_{j}=c_{j}^{\prime}+e_{j}^{\prime}-\Delta, \\
c_{k}+e_{k}=c_{k}^{\prime}+e_{k}^{\prime}+\Delta, \\
x_{N}^{\prime} P_{j}^{m_{j}} x_{N} I_{j}^{m_{j}}\left(\left(\widehat{c}, m_{j}\right),\left(c_{i}^{m^{-}}\left(\bar{x}_{i}\right), m_{i}\right)_{i \in N \backslash\{j\}}\right) P_{j}^{m_{j}}\left(\left(\widetilde{c}, m_{j}\right),\left(c_{i}^{m^{-}}\left(\bar{x}_{i}\right), m_{i}\right)_{i \in N \backslash\{j\}}\right), \\
\left(\left(\widehat{c}, m_{k}\right),\left(c_{i}^{m^{-}}\left(\bar{x}_{i}\right), m_{i}\right)_{i \in N \backslash\{k\}}\right) P_{k}^{m_{k}}\left(\left(\widetilde{c}, m_{k}\right),\left(c_{i}^{m^{-}}\left(\bar{x}_{i}\right), m_{i}\right)_{i \in N \backslash\{k\}}\right) I_{k}^{m_{k}} x_{N} P_{k}^{m_{k}} x_{N}^{\prime},
\end{gathered}
$$

with $x_{N} I_{i}^{m_{i}} x_{N}^{\prime}$ for all $i \neq j, k$; then $x_{N} \mathbf{R}(E) x_{N}^{\prime}$.

Let us now present a social ranking that satisfies these three axioms. To do so, we define the following SOF:

Social Ordering Function 1 For all $E \in \mathcal{E}$ and $x_{N}, x_{N}^{\prime} \in X^{n}$,

$$
x_{N} \mathbf{R}_{\text {lex }}^{\lambda}(E) x_{N}^{\prime} \Leftrightarrow\left(\lambda_{i}\left(x_{N}\right)\right)_{i \in N} \geq_{\operatorname{lex}}\left(\lambda_{i}\left(x_{N}^{\prime}\right)\right)_{i \in N} .
$$

$\mathbf{R}_{\text {lex }}^{\lambda}$ consists of the application of the leximin criterion $\left(\geq_{\text {lex }}\right)$ to the FHEC equal-split equivalent $\lambda_{i}\left(x_{N}\right)$. Let us make use of this function to present our result. 
Proposition 2 On the domain $\mathcal{E}, \mathbf{R}_{\mathrm{lex}}^{\lambda}$ satisfies Strong Pareto, Equal-Split Equivalent Solidarity and Equal Relative Views Solidarity.

This outcome provides a basic description of the social preferences. More precisely, Proposition 2 offers some conditions that any society that wants to implement the ranking $\mathbf{R}_{\text {lex }}^{\lambda}$ must fulfil. It should be noted that this result does not give a complete characterisation as other SOFs may also satisfy the ethical principles that we endorse. Moreover, changes in the ethical principles will not have an innocuous effect on the social preferences, and if one opts for alternative fairness axioms the ranking of allocations would have to be changed accordingly. For instance, if the axioms were focused on the amount of resources that would make the individual indifferent between the actual and the hypothetical allocations, the corresponding SOF should minimise the maximum value of such an amount. As regards the relative views, one may defend that they should not matter for equality, and that only Strong Pareto should take individual ORPs into account. Then, fairness axioms should not compensate individuals for their other-regarding views, and hence the social preferences would no longer be grounded on the value of $\lambda_{i}\left(x_{N}\right)$. In such a scenario we could study the additional properties that we should impose on ORPs in order to recover a solution that matches the one obtained in the case of self-centered preferences (see Decerf and Van der Linden 2016). Such a solution would imply maximising the minimum value of the actual proportion between $c_{i}^{m_{i}}\left(x_{i}\right)$ and $c_{i}^{m^{-}}\left(\bar{x}_{i}\right)$ (see Figure 1)

\section{Discussion}

The aim of this note is to develop a framework that allows us to make fair welfare evaluations when agents have ORPs and health is one of the dimensions of individual well-being. Additionally, and grounded on this framework, we have proposed a measure of this well-being which is defined as the individual relative view of an optimal hypothetical situation that entails the state of perfect health and the absence of externalities.

Finally, it is important to stress that other SOFs different than the one proposed here may also satisfy the principles endorsed by our society. So far, the only way to obtain complete characterisation results with ORPs is to focus on models in which agents have relative views on the average consumption alone (see Treibich 2015). The derivation of characterisation results in complex settings like the one depicted in the present paper will be part of a future research. Another issue for which our framework could be a starting point is the evaluation of the implications that ORPs might have 
on the design of an optimal income tax schedule for the provision of health care.

\section{A Appendix}

\section{A.1 Proof of Proposition 1}

For any $E \in \mathcal{E}$, let us consider an allocation $x_{N} \in X^{n}$. Because of Consumption Desirability, each individual $i \in N$ prefers a strictly positive level of consumption than no consumption at all, no matter the others' situation. Therefore, for any $c>0$ it has to be the case that $\left((c, e), x_{N \backslash\{i\}}\right) P_{i}^{m_{i}}\left(\left(0, m_{i}\right),\left(c_{j}^{m^{-}}\left(\bar{x}_{j}\right), m_{j}\right)_{j \in N \backslash\{i\}}\right) . \quad$ Hence, due to monotonicity in one's own consumption the FHEC equal-split equivalent $\lambda_{i}\left(x_{N}\right)$ cannot be negative.

As regards the existence of $\lambda_{i}\left(x_{N}\right)$, due to the previous result, together with continuity and monotonicity, there exists $\widehat{\lambda} \in \mathbb{R}_{+}$such that for all $\lambda \leq \widehat{\lambda}$ we have that $x_{N} R_{i}^{m_{i}}\left(\left(\lambda c_{i}^{m^{-}}\left(\bar{x}_{i}\right), m_{i}\right),\left(c_{j}^{m^{-}}\left(\bar{x}_{j}\right), m_{j}\right)_{j \in N \backslash\{i\}}\right)$. With respect to the situations that are preferred to $x_{N}$, for some extreme versions of altruism and egoism there may be some allocations for which no $\lambda \in \mathbb{R}_{++}$ guarantees that $\left(\left(\lambda c_{i}^{m^{-}}\left(\bar{x}_{i}\right), m_{i}\right),\left(c_{j}^{m^{-}}\left(\bar{x}_{j}\right), m_{j}\right)_{j \in N \backslash\{i\}}\right) P_{i}^{m_{i}}\left(x_{i}, x_{N \backslash\{i\}}\right)$. Specifically, if $x_{N \backslash\{i\}}$ is sufficiently small (respectively, large) an extremely egoistic (altruistic) individual may be so well-off that $\lambda_{i}\left(x_{N}\right)$ might go to $+\infty$. Nevertheless, due to Plurality of Preferences there exists a set of individuals $N^{S} \subset N$ who have self-centered preferences. For such agents it is always possible to find a value $\widetilde{\lambda} \in \mathbb{R}_{++}$such that, by monotonicity, for all $\lambda \geq \tilde{\lambda}$ we have that $\left(\left(\lambda c_{i}^{m^{-}}\left(\bar{x}_{i}\right), m_{i}\right),\left(c_{j}^{m^{-}}\left(\bar{x}_{j}\right), m_{j}\right)_{j \in N \backslash\{i\}}\right) R_{i}^{m_{i}} x_{N}$. Additionally, among the agents who have altruistic preferences, $N^{A} \subset N$, the value of $\widetilde{\lambda}$ can also be defined for those who do not exhibit extreme relative views, a group that we denote by $N_{1}^{A} \subseteq N^{A}$. The same happens for those who have egoistic preferences, that is, $N_{1}^{E} \subseteq N^{E} \subset N$. As a result of this, for any $i \in N_{1}=N^{S} \cup N_{1}^{A} \cup N_{1}^{E} \neq \emptyset$ this critical value $\tilde{\lambda}$ is well-defined. Hence, for any $i \in N_{1}$ both values $\widehat{\lambda}$ and $\widetilde{\lambda}$ exist, and given that $\mathbb{R}_{+}$is a connected space, by continuity there exits $\lambda_{i}\left(x_{N}\right) \in \mathbb{R}_{+}$such that $x_{N} I_{i}^{m_{i}}\left(\left(\lambda_{i}\left(x_{N}\right) c_{i}^{m^{-}}\left(\bar{x}_{i}\right), m_{i}\right),\left(c_{j}^{m^{-}}\left(\bar{x}_{j}\right), m_{j}\right)_{j \in N \backslash\{i\}}\right)$.

Finally, let us assume that for any individual $i \in N_{1}$ there exist $\lambda_{i}\left(x_{N}\right), \lambda_{i}^{\prime}\left(x_{N}\right) \in \mathbb{R}_{+}$such that $\lambda_{i}\left(x_{N}\right)>\lambda_{i}^{\prime}\left(x_{N}\right)$. Because of monotonicity in one's own consumption $\left(\left(\lambda_{i}\left(x_{N}\right) c_{i}^{m^{-}}\left(\bar{x}_{i}\right), m_{i}\right),\left(c_{j}^{m^{-}}\left(\bar{x}_{j}\right), m_{j}\right)_{j \in N \backslash\{i\}}\right) P_{i}^{m_{i}}$ 
$\left(\left(\lambda_{i}^{\prime}\left(x_{N}\right) c_{i}^{m^{-}}\left(\bar{x}_{i}\right), m_{i}\right),\left(c_{j}^{m^{-}}\left(\bar{x}_{j}\right), m_{j}\right)_{j \in N \backslash\{i\}}\right)$. That is, the individual is not indifferent between the two previous allocations, and hence $\lambda_{i}\left(x_{N}\right)$ and $\lambda_{i}^{\prime}\left(x_{N}\right)$ cannot both at the same time represent her preferences over the allocation $x_{N} \in X^{n}$.

Consequently, the smallest $\lambda_{i}\left(x_{N}\right)$ in the economy is well-defined, that is, this value exists and it is positive and unique.

\section{A.2 Proof of Proposition 2}

For any $E \in \mathcal{E}$, let us consider $x_{N}, x_{N}^{\prime} \in X^{n}$ such that $x_{N} R_{i}^{m_{i}} x_{N}^{\prime}$. Hence, $\left(\left(\lambda_{i}\left(x_{N}\right) c_{i}^{m^{-}}\left(\bar{x}_{i}\right), m_{i}\right),\left(c_{j}^{m^{-}}\left(\bar{x}_{j}\right), m_{j}\right)_{j \in N \backslash\{i\}}\right) R_{i}^{m_{i}}\left(\left(\lambda_{i}\left(x_{N}^{\prime}\right) c_{i}^{m^{-}}\left(\bar{x}_{i}\right), m_{i}\right),\left(c_{j}^{m^{-}}\left(\bar{x}_{j}\right), m_{j}\right)_{j \in N \backslash\{i\}}\right)$. By reflexivity and strict monotonicity in one's own consumption we can conclude that $\lambda_{i}\left(x_{N}\right) \geq \lambda_{i}\left(x_{N}^{\prime}\right)$, and hence the FHEC equal-split equivalent $\lambda_{i}\left(x_{N}\right)$ is a valid representation of $R_{i}^{m_{i}}$.

We now show that $\mathbf{R}_{\text {lex }}^{\lambda}$ satisfies the axioms that our society endorses.

- Strong Pareto: let us take $x_{N}, x_{N}^{\prime} \in X^{n}$ with $x_{N} R_{i}^{m_{i}} x_{N}^{\prime}$ for all $i \in N$. Since $\lambda_{i}\left(x_{N}\right)$ represents $R_{i}^{m_{i}}$ it follows that $\min _{i \in N} \lambda_{i}\left(x_{N}\right) \geq \min _{i \in N} \lambda_{i}\left(x_{N}^{\prime}\right)$, and hence according to $\mathbf{R}_{\text {lex }}^{\lambda}$ we have that $x_{N} \mathbf{R}(E) x_{N}^{\prime}$. If there exists $j \in N$ such that $x_{N} P_{j}^{m_{j}} x_{N}^{\prime}$ then $\lambda_{j}\left(x_{N}\right)>\lambda_{j}\left(x_{N}^{\prime}\right)$. Therefore, $x_{N} \mathbf{P}(E) x_{N}^{\prime}$.

- Equal-Split Equivalent Solidarity: let us take $\Delta \in \mathbb{R}$ and $x_{N}, x_{N}^{\prime} \in X^{n}$ in which there exist $j, k \in N$ such that $c_{j}+e_{j}=c_{j}^{\prime}+e_{j}^{\prime}-\Delta, c_{k}+e_{k}=c_{k}^{\prime}+$ $e_{k}^{\prime}+\Delta, x_{N}^{\prime} P_{j}^{m_{j}} x_{N} P_{j}^{m_{j}}\left(c_{i}^{m^{-}}\left(\bar{x}_{i}\right), m_{i}\right)_{i \in N}$ and $\left(c_{i}^{m^{-}}\left(\bar{x}_{i}\right), m_{i}\right)_{i \in N} P_{k}^{m_{k}} x_{N} P_{k}^{m_{k}} x_{N}^{\prime}$. By definition $\lambda_{i}\left(\left(c_{i}^{m^{-}}\left(\bar{x}_{i}\right), m_{i}\right)_{i \in N}\right)=1$ for all $i \in N$, which implies that $\lambda_{j}\left(x_{N}^{\prime}\right)>\lambda_{j}\left(x_{N}\right)>1>\lambda_{k}\left(x_{N}\right)>\lambda_{k}\left(x_{N}^{\prime}\right)$. Moreover, $\lambda_{i}\left(x_{N}\right)=\lambda_{i}\left(x_{N}^{\prime}\right)$ for all $i \neq j, k$, and hence according to $\mathbf{R}_{\text {lex }}^{\lambda}$ we have that $x_{N} \mathbf{R}(E) x_{N}^{\prime}$.

- Equal Relative Views Solidarity: let us consider $\Delta \in \mathbb{R}$ and $x_{N}, x_{N}^{\prime} \in$ $X^{n}$ in which there exist individuals $j, k \in N$ with $R_{j}^{m}=R_{k}^{m}$ for all $m \in \mathcal{M}$ such that $c_{j}+e_{j}=c_{j}^{\prime}+e_{j}^{\prime}-\Delta$ and $c_{k}+e_{k}=c_{k}^{\prime}+e_{k}^{\prime}+\Delta$. Moreover, there exists $\widehat{c}, \widetilde{c} \in \mathbb{R}_{++}$such that the following preference relations are satisfied:

$x_{N}^{\prime} P_{j}^{m_{j}} x_{N} I_{j}^{m_{j}}\left(\left(\widehat{c}, m_{j}\right),\left(c_{i}^{m^{-}}\left(\bar{x}_{i}\right), m_{i}\right)_{i \in N \backslash\{j\}}\right) P_{j}^{m_{j}}\left(\left(\widetilde{c}, m_{j}\right),\left(c_{i}^{m^{-}}\left(\bar{x}_{i}\right), m_{i}\right)_{i \in N \backslash\{j\}}\right)$, $\left(\left(\widehat{c}, m_{k}\right),\left(c_{i}^{m^{-}}\left(\bar{x}_{i}\right), m_{i}\right)_{i \in N \backslash\{k\}}\right) P_{k}^{m_{k}}\left(\left(\widetilde{c}, m_{k}\right),\left(c_{i}^{m^{-}}\left(\bar{x}_{i}\right), m_{i}\right)_{i \in N \backslash\{k\}}\right) I_{k}^{m_{k}} x_{N} P_{k}^{m_{k}} x_{N}^{\prime}$,

with $x_{N} I_{i}^{m_{i}} x_{N}^{\prime}$ for any $i \neq j, k$. Given that the FHEC equal-split equivalent is a valid measure of $R_{i}^{m_{i}}$ we can establish that:

$\lambda_{j}\left(\left(\widehat{c}, m_{j}\right),\left(c_{i}^{m^{-}}\left(\bar{x}_{i}\right), m_{i}\right)_{i \in N \backslash\{j\}}\right)=\frac{\widehat{c}}{c_{j}^{m^{-}}\left(\bar{x}_{j}\right)}=\frac{\widehat{c}}{c_{k}^{m-}\left(\bar{x}_{k}\right)}=\lambda_{k}\left(\left(\widehat{c}, m_{k}\right),\left(c_{i}^{m^{-}}\left(\bar{x}_{i}\right), m_{i}\right)_{i \in N \backslash\{k\}}\right)$,
$\lambda_{j}\left(\left(\widetilde{c}, m_{j}\right),\left(c_{i}^{m^{-}}\left(\bar{x}_{i}\right), m_{i}\right)_{i \in N \backslash\{j\}}\right)=\frac{\widetilde{c}}{c_{j}^{m-}\left(\bar{x}_{j}\right)}=\frac{\widetilde{c}}{c_{k}^{m-}\left(\bar{x}_{k}\right)}=\lambda_{k}\left(\left(\widetilde{c}, m_{k}\right),\left(c_{i}^{m^{-}}\left(\bar{x}_{i}\right), m_{i}\right)_{i \in N \backslash\{k\}}\right)$. 
These equalities hold because $j$ and $k$ share the same relative views, which implies that $c_{j}^{m^{-}}\left(\bar{x}_{j}\right)=c_{k}^{m^{-}}\left(\bar{x}_{k}\right)$. As a result of this we have that $\lambda_{j}\left(x_{N}^{\prime}\right)>$ $\lambda_{j}\left(x_{N}\right)>\lambda_{k}\left(x_{N}\right)>\lambda_{k}\left(x_{N}^{\prime}\right)$. Moreover, since the rest of the agents must remain indifferent between the two allocations, it must be the case that $\lambda_{i}\left(x_{N}\right)=\lambda_{i}\left(x_{N}^{\prime}\right)$ for all $i \neq j, k$. Consequently, according to $\mathbf{R}_{\text {lex }}^{\lambda}$ we conclude that $x_{N} \mathbf{R}(E) x_{N}^{\prime}$.

\section{References}

[1] Braakmann N (2014): The consequences of own and spousal disability on labor market outcomes and subjective well-being: evidence from Germany. Review of Economics of the Household 12: 717-736.

[2] Bruhin A, Winkelmann R (2009): Happiness functions with preference interdependence and heterogeneity: the case of altruism within the family. Journal of Population Economics 22: 1063-1080.

[3] Decerf B, Van der Linden M (2016): Fair social orderings with otherregarding preferences. Social Choice and Welfare 46: 655-694.

[4] Fehr E, Schmidt K M (2006): The economics of fairness, reciprocity and altruism - Experimental evidence and new theories. In S C Kolm, J M Ythier (eds.), Handbook of the Economics of Giving, Altruism and Reciprocity. North-Holland: 615-691.

[5] Fleurbaey M (2005): Health, wealth, and fairness. Journal of Public Economic Theory 2: 253-284.

[6] Fleurbaey M (2012): The importance of what people care about. Politics, Philosophy \& Economics 11: 415-447.

[7] Fleurbaey M, Maniquet F (2011): A theory of fairness and social welfare. Cambridge University Press.

[8] Fleurbaey M, Schokkaert E (2011): Equity in health and health care. In M V Pauly, T G Mcguire, P P Barros (eds.), Handbook of Health Economics 2. North-Holland: 1003-1092.

[9] Kerschbamer R, Sutter M, Dulleck U (2016): How social preferences shape incentives in (experimental) markets for credence goods. The Economic Journal. DOI: 10.1111/ecoj.12284. 
[10] Treibich R (2015): Welfare egalitarianism with other-regarding preferences. Mimeo. University of Southern Denmark.

[11] Valletta G (2014): Health, fairness and taxation. Social Choice and Welfare 43: 101-140. 